\title{
Effects of Rikkunshito (TJ-43) on Esophageal Motor Function and Gastroesophageal Reflux
}

\author{
Terumi Morita, ${ }^{1}$ Kenji Furuta, ${ }^{1 *}$ Kyoichi Adachi, ${ }^{2}$ Shunji Ohara, ${ }^{1}$ Takashi Tanimura, ${ }^{1}$ Kenji Koshino, ${ }^{1}$ Tomochika Uemura, ${ }^{3}$ Kohj \\ Naora $^{3}$ and Yoshikazu Kinoshita ${ }^{1}$ \\ Second Departments of ${ }^{1}$ Internal Medicine and ${ }^{2}$ Clinical Nursing, Shimane University Faculty of Medicine, Izumo-shi, Shimane, Japan; and \\ ${ }^{3}$ Department of Pharmacy, Shimane University Hospital, Izumo-shi, Shimane, Japan
}

\begin{abstract}
Background/Aims
Rikkunshito (TJ-43), an herbal medicine, has been demonstrated to relieve gastroesophageal reflux symptoms. However, the effects of TJ-43 on esophageal motor functions have not been fully determined. This double-blind crossover study was performed to investigate the effects of TJ-43 on esophageal motor functions and gastroesophageal reflux.
\end{abstract}

\section{Methods}

The subjects were 10 normal male volunteers. Lower esophageal sphincter pressure and esophageal body peristaltic contractions with and without 1-week administration of TJ-43 were examined in a crossover fashion. Post-prandial gastroesophageal reflux was also determined using a multi-channel impedance pH dual monitor.

\section{Results}

TJ-43 at a standard dose of $7.5 \mathrm{~g} /$ day did not significantly augment esophageal peristaltic contraction pressure measured in the proximal, middle and distal segments of the esophagus, whereas increment of resting lower esophageal sphincter pressure was observed in a supine position. In addition, TJ-43 administration did not decrease post-prandial gastroesophageal acid, non-acid reflux events or accelerate esophageal clearance time.

\section{Conclusions}

TJ-43 at a standard dose did not have a significant effect on esophageal motor activity or gastroesophageal reflux in healthy adults.

(J Neurogastroenterol Motil 2012;18:181-186)

Key Words

Herbal medicine; Esophageal pH monitoring; Esophageal sphincter, lower; Peristalsis

\section{Introduction}

Gastroesophageal reflux disease (GERD) is a disease frequently encountered in gastroenterology clinics in both western countries and Japan. ${ }^{1-4}$ Decreased esophageal motor function is one of the most important factors for occurrence of GERD, ${ }^{5}$ thus, prokinetic agents that increase lower esophageal sphincter

Received: November 26, 2011 Revised: January 25, 2012 Accepted: January 30, 2012

(c) This is an Open Access article distributed under the terms of the Creative Commons Attribution Non-Commercial License (http://creativecommons. org/licenses/by-nc/3.0) which permits unrestricted non-commercial use, distribution, and reproduction in any medium, provided the original work is properly cited.

*Correspondence: Kenji Furuta, MD, PhD

Second Department of Internal Medicine, Shimane University Faculty of Medicine, 89-1 Enya-cho, Izumo-shi, Shimane 693-8501, Japan

Tel: +81-853-20-2190, Fax: +81-853-20-2187, E-mail: kfuruta@med shimane-u.ac.jp

Financial support: None.

Conflicts of interest: None. 
(LES) pressure and esophageal body contraction are considered to be effective for treatment of GERD patients. The powder extract of the herbal medicine, rikkunshito, has been reported to be effective in improving various upper gastro-intestinal symptoms, and is widely administrated to patients with GERD and functional dyspepsia (FD) in Japan. ${ }^{6-10}$ Rikkunshito, extracted from a mixture of Atractylodis Lanceae Rhizoma, Ginseng Radix, Pinelliae Tuber, Hoelen, Zizyphi Fructus, Aurantii Nobilis Percarpium, Glycyrrhizae Radix and Zingiberis Rhizoma, is manufactured and distributed as TJ-43 by Tsumura Co. (Tokyo, Japan). ${ }^{11}$ Several studies have demonstrated that TJ-43 stimulates gastric motor activities and accelerate gastric emptying. ${ }^{7,10,12}$ However, the effects of TJ-43 on esophageal motor functions have not been fully examined, though it was reported to be effective for relief of reflux symptoms, such as heartburn and acid regurgitation. ${ }^{6,7,9,13}$ We conducted the present double-blind crossover study to clarify the effects of TJ-43 on esophageal motor functions and gastroesophageal reflux events.

\section{Materials and Methods}

We studied 10 healthy male volunteers $(22.8 \pm 2.0$ years old) who had no history of gastrointestinal surgery and received no medication which might have affected gastroesophageal function.

Esophageal motor function and post-prandial gastroesophageal reflux were determined after a 7-day administration of $\mathrm{TJ}-43$ or a placebo in random order using a double-blind crossover design. TJ-43 at $2.5 \mathrm{~g}$ was administrated 3 times per day (commonly used for adult patients in Japan with GERD and FD symptoms) 30 minutes before each meal. Lactose was used as the placebo drug. TJ-43 or the placebo was placed in identical capsules by 2 pharmacists (Tomochika Uemura and Kohji Naora) to conceal the contents, and the contents of the capsules were unknown to the examiners of esophageal motor function and post-prandial gastroesophageal reflux.

All subjects were tested after 7-day administrations of TJ-43 and the placebo, with at least 1-week interval between. On the last day of administration, determinations of esophageal motor function and post-prandial gastroesophageal reflux were performed. We examined esophageal motor function using high-resolution 36-channel manometry (ManoScan ${ }^{360 \mathrm{TM}}$; Sierra Scientific Instruments, Los Angeles, CA, USA). ${ }^{14-16}$ Briefly, prior to each measurement, the transducers were calibrated according to the manufacturer's instructions, then a manometric catheter was in- serted transnasally using a small amount of $2 \%$ lidocaine jelly (Xylocaine jelly; AstraZeneka Co., Osaka, Japan). The measurements were started 5 minutes after insertion for stabilization with the subject in a sitting position. Initially, we determined resting LES pressure for at least 5 minutes, then measured esophageal body peristaltic contractions after the subject drank $5 \mathrm{~mL}$ of water. That, was repeated at intervals of 2 minutes until we obtained 5 complete contraction records. After finishing the determination of esophageal motor activity in a sitting position, the intraesophageal pressure measurements were repeated in the same manner with the subject in a supine position. For analysis, the esophageal body peristaltic contractions were divided into 3 segments from segments 1 to 3 (proximal, middle and distal) by 2 contraction troughs, as reported previously. ${ }^{17-20}$ The maximum peristaltic contractions in each segment were determined, then the mean of 5 individual contractions was calculated as used as the contraction value for each of the segments in each subject.

Following the esophageal motor function tests, we utilized a composite 6-channel impedance and 2-channel $\mathrm{pH}$ sensor catheter (Sandhill Scientific Inc., Highlands Ranch, CO, USA) that was inserted transnasally after calibration with a buffer solution at $\mathrm{pH} 4.0$ and 7.0, according to the manufacturer's instructions. We placed the impedance sensors at 3, 5, 7, 9, 15 and $17 \mathrm{~cm}$ above the LES, and the esophageal $\mathrm{pH}$ at $5 \mathrm{~cm}$ above the LES. One hour after insertion of the catheter for impedance and $\mathrm{pH}$ determination, a standardized 1,617 kcal high caloric meal was supplied and consumed within 20 minutes. This high caloric meal consisted of a large plate of rice and curry, as well as cheese and tuna soup (S\&B Foods Inc., Tokyo; Hagoromo Foods Company, Shizuoka; and Ajinomoto Company, Tokyo, Japan), and contained $49.7 \mathrm{~g}$ of protein, $220.3 \mathrm{~g}$ of carbohydrates and $59.8 \mathrm{~g}$ of fat. Data collection using a multichannel intraluminal impedance-pH monitoring system (Sleuth ${ }^{\mathrm{TM}}$; Sandhill Scientific Inc.) was performed during the 3-hour period after the meal. We then utilized a computerized algorithm (Autoscan; Sandhill Scientific Inc.) to identify reflux episodes as changes in impedance from the distal to proximal esophagus over time. The tracings thus obtained were manually reviewed to confirm that the reflux episodes were correctly identified by the automated algorithm. Reflux episodes were classified as follows: (1) liquid only (decrease in impedance progressing proximally), (2) gas only (rise in impedance above $3,000 \Omega$ in at least 2 channels progressing proximally) and (3) mixed (gas-liquid: a combination of liquid and gas impedance patterns). ${ }^{21-23}$ We then further analyzed the liquid only and mixed reflux episodes to determine the 

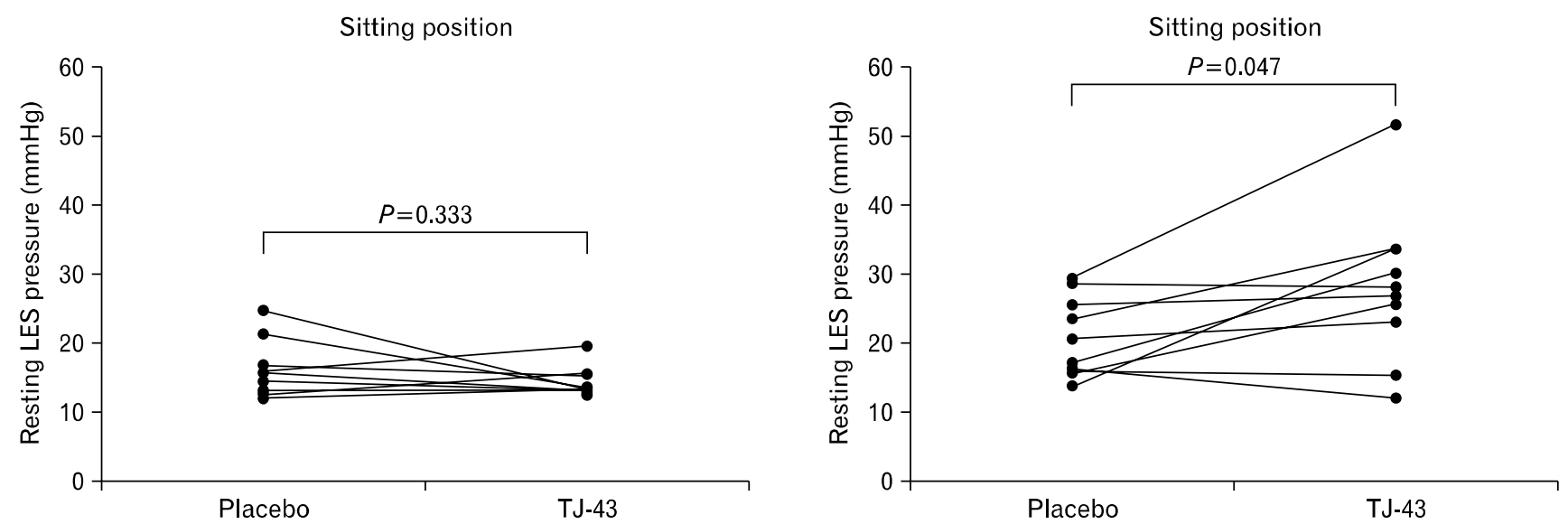

Figure 1. Resting lower esophageal sphincter (LES) pressure in sitting and supine positions determined during TJ-43 and placebo administrations. LES pressure was higher during TJ-43 administration in the supine position, though the difference was not significant.

acidity of the refluxes. Finally, reflux episodes were classified as: (1) acidic, for those with a nadir $\mathrm{pH}$ recorded during a mutichannel intraluminal impedance-detected reflux pattern that was less than 4.0 ; (2) weakly acidic, when $\mathrm{pH}$ declined more than 1.0 and the nadir was between 4.0 and 7.0 ; and (3) non-acidic, in instances when the $\mathrm{pH}$ nadir was greater than 7.0. ${ }^{23}$

A Wilcoxon signed rank test was used to compare paired data. All calculations were done with Stat View 5.0 software (Abacus Concepts Inc., Berkeley, CA, USA) for Macintosh. Data were expressed as the mean $\pm \mathrm{SE}$. A difference level of $P<$ 0.05 was considered to be significant.

This study was performed according to the declaration of Helsinki and the study protocol was approved by the ethical committee of Shimane University Faculty of Medicine. Written informed consent was obtained from each of the study subjects before enrollment.

\section{Results}

Resting LES pressures in the sitting and supine positions during administration of TJ-43 or the placebo are shown in Figure 1. LES pressure determined in the supine position was significantly greater than when measured in the sitting position, as previously reported. ${ }^{19,20,24}$ There was no statistically significant difference in LES pressure values determined during the administrations of TJ-43 and the placebo in the sitting position (14.4 \pm 0.7 vs $16.3 \pm 1.3 \mathrm{mmHg} ; P=0.798)$, whereas those in the supine position were significantly different between them $(28.0 \pm$ 3.5 vs $20.6 \pm 1.8 \mathrm{mmHg} ; P=0.047$ ). Esophageal body peristaltic contractions measured at each segment 1,2 and 3 (proximal, middle and distal) of the esophagus in the sitting and supine positions are presented in Figure 2. In the 2 positions, the distal segment (segment 3) had the greatest peak contraction pressure. In addition, contraction pressure was greater in the supine position for each segment. TJ-43 administration did not enhance the esophageal peristaltic contraction pressure in any in either the sitting or supine position.

There was no statistically significant difference between the number of total post-prandial 3-hour reflux episodes during the TJ-43 and placebo administrations, though the number of reflux episodes tended to be higher during TJ-43 administration (43.1 $\pm 8.0 / 3$ hours vs $34.2 \pm 6.0 / 3$ hours; $P=0.308$ ). During the administration of $\mathrm{TJ}-43$, the refluxate consisted of liquid only (71.7\%), gas only $(4.4 \%)$ and mixed $(23.9 \%)$ reflux, while with the placebo the refluxate consisted of liquid only (79.8\%), gas only $(4.1 \%)$ and mixed $(16.1 \%)$ reflux. Liquid only was the most frequently observed type of reflux in each post-prandial period, followed by mixed (Fig. 3). There were no significant differences for the ratios of weakly-acid and non-acid reflux between liquid only and mixed reflux events during administration with TJ-43 and with the placebo. The percentage times at intraesophageal $\mathrm{pH}<4.0$ during the 3 -hour post-prandial period are shown in Figure 4. There were no significant differences between the TJ-43 and placebo administrations regarding the percentage of esophageal acid ( $\mathrm{pH}<4.0$ ) exposure time during the 3-hour post-prandial period $(6.5 \pm 2.0 \%$ vs $5.8 \pm 1.3 \% ; P>0.999)$. On the other hand, the bolus clearance time of the gastroesophageal refluxate tended to be shorter during TJ-43 administration $(25.9 \pm 4.3$ vs $37.0 \pm 8.8$ seconds; $P=0.139)$. 
Segments 1 (proximal)
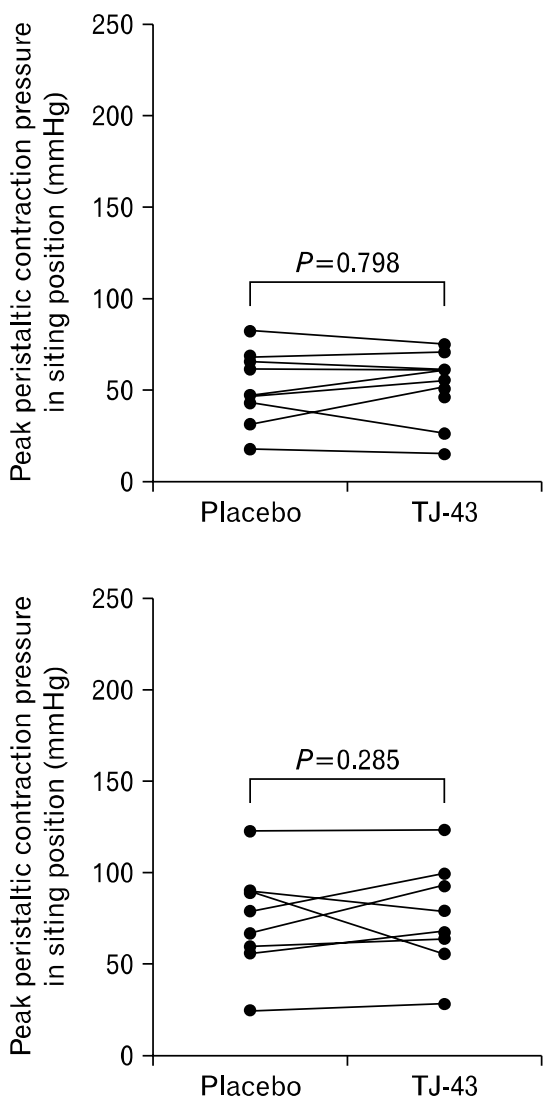

Segments 2 (proximal)
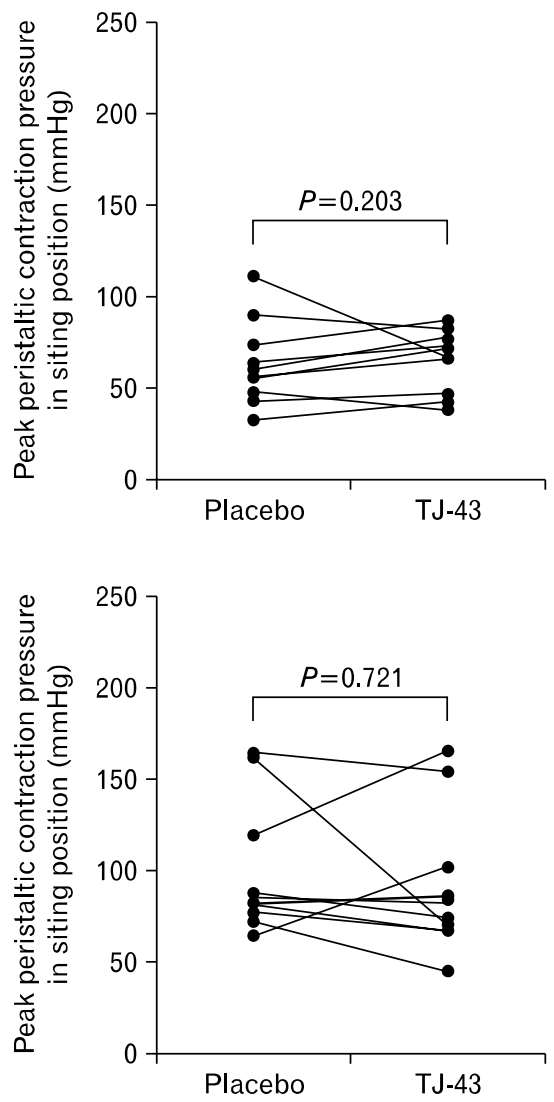

Segments 3 (proximal)
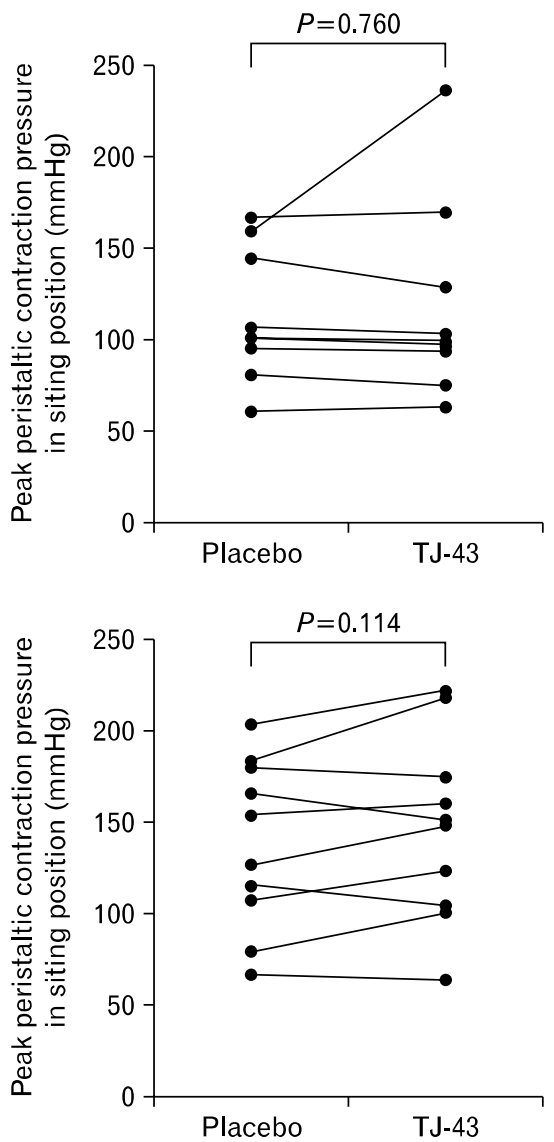

Figure 2. Peak peristaltic contraction pressures in sitting and supine positions in segments 1 (A), 2 (B) and 3 (C). The distal segment (segment 3) had a higher level of peristaltic pressure than the proximal segment (segment 1 ) in both the sitting and supine positions. There was no statistically significant difference in regard to peristaltic pressure between the TJ-43 and placebo administration periods in the sitting and supine positions.

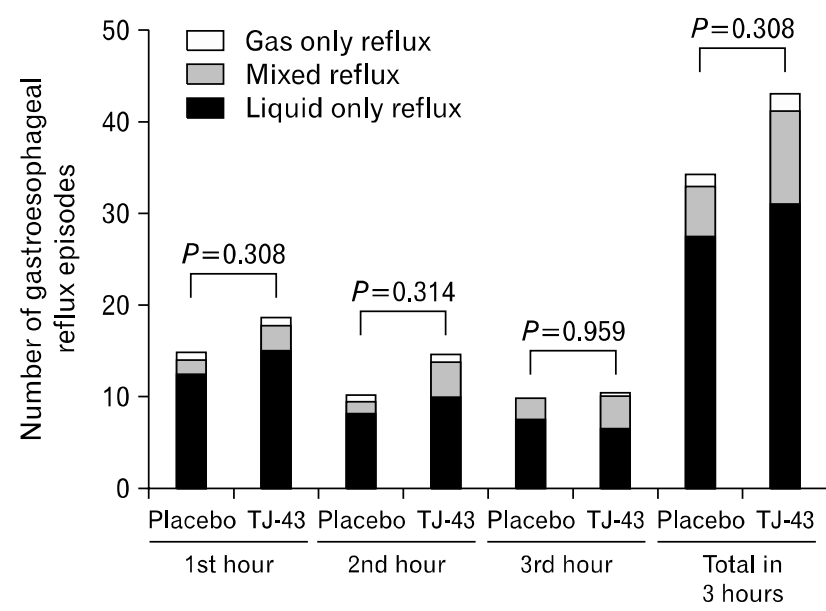

Figure 3. Mean post-prandial gastroesophageal reflux episodes, as determined by multi-channel impedance $\mathrm{pH}$ monitoring. The 3-hour post-prandial period was divided into three 1-hour periods. Liquid containing reflux (liquid only and mixed) was the major type observed during both the placebo and $\mathrm{TJ}-43$ administrations.

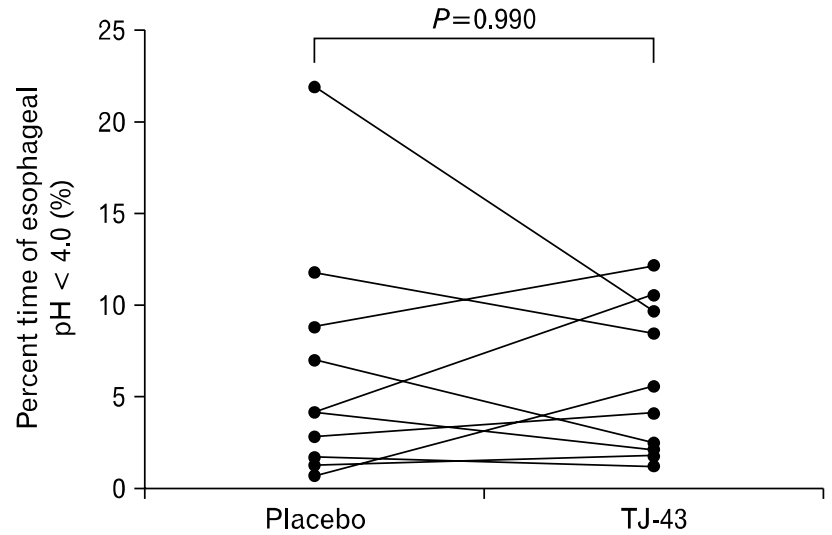

Figure 4. Percentage of time at $\mathrm{pH}$ lower than 4 during the 3-hour post-prandial period. TJ-43 did not significantly change esophageal acid exposure time. 


\section{Discussion}

Incompetence of the LES, decrease in esophageal motor function, reduced salivary secretion, and delayed gastric emptying has been observed in GERD patients, while prokinetic agents are expected to improve those anti-reflux functions. ${ }^{1}$ Indeed, administration of cisapride was demonstrated to augment LES pressure, esophageal body contraction, salivary secretion and gastric emptying, and also reported to effectively control reflux symptoms in patients with GERD. ${ }^{25-29}$ Although cisapride has been used for treatment of GERD and shown to be equally effective as a histamine 2 receptor antagonist, ${ }^{27}$ it was later withdrawn from the market because of the cardiac side effects. ${ }^{30}$ At present, several other prokinetic agents are used for treatment of patients with various upper gastrointestinal symptoms, and their administration has been reported to be effective in patients with $\mathrm{FD}$ as well as with GERD.

TJ-43 is an herbal medicine reported to be effective for several upper gastro-intestinal symptoms, and widely administrated in Japan to patients with GERD and FD. ${ }^{7,810,11,13} \mathrm{TJ}-43$ has also been reported to stimulate gastric motor activities and accelerate gastric emptying. ${ }^{710-12}$ Improvement in esophageal acid clearance was also demonstrated following administration in pediatric patients with GERD. ${ }^{9}$ However, the influence of TJ-43 on esophageal motor function and gastroesophageal reflux events in adults has not been fully investigated.

In the present study, we conducted a detailed investigation of the effects of TJ-43 at a standard dose on esophageal motor activity and gastroesophageal acid/non-acid reflux. However, we did not find evidence of its efficacy for anti-reflux functions. On the other hand, TJ-43 elevated resting LES pressure in a supine position, and tended to accelerate esophageal bolus clearance and increase post-prandial reflux, though that latter finding is contrary to a previous report regarding the effect on esophageal motor function in pediatric GERD patients. ${ }^{9}$ We employed a placebo-controlled double blind design in the present study, and also utilized highly sensitive and reliable methods to determine esophageal motor activity and gastroesophageal reflux. Therefore, the efficacy of TJ-43 for adult patients with GERD may not be related to augmentation of esophageal motor function, while increased gastric motor function by administration of $\mathrm{TJ}-43$ may relieve GERD symptoms. Investigation of the effects of TJ-43 on esophageal sensory and perception mechanisms is important in a future study.
Our study has some limitations. We administrated TJ-43 to healthy young volunteers and not GERD patients with reduced esophageal motor function and frequent gastro-esophageal reflux events, thus we did not evaluate the efficacy of TJ-43 on GERD symptoms. Additional investigations of patients with GERD are necessary to confirm the effects of TJ-43 on esophageal motor function.

In conclusion, this double-blind crossover study did not reveal a significant effect of $\mathrm{TJ}-43$ on esophageal motor activity or gastroesophageal reflux events. Future studies are needed to clarify its effects in patients with GERD.

\section{References}

1. Furukawa N, Iwakiri R, Koyama T, et al. Proportion of reflux esophagitis in 6010 Japanese adults: prospective evaluation by endoscopy. J Gastroenterol 1999;34:441-444.

2. Shimazu T, Matsui T, Furukawa K, et al. A prospective study of the prevalence of gastroesophageal reflux disease and confounding factors. J Gastroenterol 2005;40:866-872.

3. Mishima I, Adachi K, Arima N, et al. Prevalence of endoscopically negative and positive gastroesophageal reflux disease in the Japanese. Scand J Gastroenterol 2005;40:1005-1009.

4. Fujiwara Y, Arakawa T. Epidemiology and clinical characteristics of GERD in the Japanese population. J Gastroenterol 2009;44:518534.

5. Dodds WJ, Hogan WJ, Helm JF, Dent J. Pathogenesis of reflux esophagitis. Gastroenterology 1981;81:376-394.

6. Koide A, Yamaguchi T, Koyama H, et al. Effect and role of TJ-43: Rikkun-shi-to from the aspects of endoscopic findings and QOL improvement in GERD patients. Gastroenterology 2005;128:A530.

7. Tatsuta M, Iishi H. Effect of treatment with liu-jun-zi-tang (TJ-43) on gastric emptying and gastrointestinal symptoms in dyspeptic patients. Aliment Pharmacol Ther 1993;7:459-462.

8. Takeda H, Sadakane C, Hattori T, et al. Rikkunshito, an herbal medicine, suppresses cisplatin-induced anorexia in rats via 5-HT2 receptor antagonism. Gastroenterology 2008;134:2004-2013.

9. Kawahara H, Kubota A, Hasegawa T, et al. Effects of rikkunshito on the clinical symptoms and esophageal acid exposure in children with symptomatic gastroesophageal reflux. Pediatr Surg Int 2007;23: 1001-1005.

10. Kawahara H, Mitani Y, Nomura M, et al. Impact of rikkunshito, an herbal medicine, on delayed gastric emptying in profoundly handicapped patients. Pediatr Surg Int 2009;25:987-990.

11. Yagi M, Homma S, Kubota M, et al. The herbal medicine Rikkunshito stimulates and coordinates the gastric myoelectric activity in post-operative dyspeptic children after gastrointestinal surgery. Pediatr Surg Int 2004;19:760-765.

12. Hayakawa T, Arakawa T, Kase Y, et al. Liu-Jun-Zi-Tang, a kampo medicine, promotes adaptive relaxation in isolated guinea pig stomachs. Drugs Exp Clin Res 1999;25:211-218.

13. Hiyama T, Yoshihara M, Tanaka S, Haruma K, Chayama K. Strategy for treatment of nonerosive reflux disease in Asia. World J 
Gastroenterol 2008;28:3123-3128.

14. Fox MR, Bredenoord AJ. Oesophageal high-resolution manometry: moving from research into clinical practice. Gut 2008;57:405-423.

15. Park MI. Clinical usefulness of high-resolution manometry. Korean J Neurogastroenterol Motil 2009;15:107-115.

16. Salvador R, Dubecz A, Polomsky M, et al. A new era in esophageal diagnostics: the image-based paradigm of high-resolution manometry. J Am Coll Surg 2009;208:1035-1044.

17. Clouse RE, Staiano A. Topography of the esophageal peristaltic pressure wave. Am J Physiol 1991;261(4 Pt 1):G677-G684.

18. Clouse RE, Staiano A. Topography of normal and high-amplitude esophageal peristalsis. Am J Physiol 1993;265(6 Pt 1):G1098-G1107.

19. Yoshida K, Furuta K, Adachi K, et al. Effects of anti-hypertensive drugs on esophageal body contraction. World J Gastroenterol 2010; 16:987-991.

20. Koshino K, Adachi K, Furuta K, et al. Effects of mosapride on esophageal functions and gastroesophageal reflux. J Gastroenterol Hepatol 2010;25:1066-1071.

21. Shay S, Tutuian R, Sifrim D, et al. Twenty-four hour ambulatory simultaneous impedance and $\mathrm{pH}$ monitoring: a multicenter report of normal values from 60 healthy volunteers. Am J Gastroenterol 2004; 99:1037-1043.

22. Vela MF, Camacho-Lobato L, Srinivasan R, Tutuian R, Katz PO, Castell DO. Simultaneous intraesophageal impedance and $\mathrm{pH}$ measurement of acid and nonacid gastroesophageal reflux: effect of omeprazole. Gastroenterology 2001;120:1599-1606.

23. Sifrim D, Castell D, Dent J, Kahrilas PJ. Gastro-oesophageal reflux monitoring: review and consensus report on detection and definitions of acid, non-acid, and gas reflux. Gut 2004;53:1024-1031.

24. Iwakiri K, Sugiura T, Kotoyori M, et al. Effect of body position on lower esophageal sphincter pressure. J Gastroenterol 1999;34:305309.

25. Cucchiara S, Staiano A, Boccieri A, et al. Effects of cisapride on parameters of oesophageal motility and on the prolonged intraoesophageal $\mathrm{pH}$ test in infants with gastro-oesophageal reflux disease. Gut 1990;31:21-25.

26. Galmiche JP, Brandstatter G, Evreux M, et al. Combined therapy with cisapride and cimetidine in severe reflux oesophagitis: a double blind controlled trial. Gut 1988;29:675-681.

27. Geldof H, Hazelhoff B, Otten MH. Two different dose regimens of cisapride in the treatment of reflux oesophagitis: a double-blind comparison with ranitidine. Aliment Pharmacol Ther 1993;7:409-415.

28. Goldin GF, Marcinkiewicz M, Zbroch T, Bityutskiy LP, McCallum RW, Sarosiek J. Esophagoprotective potential of cisapride. An additional benefit for gastroesophageal reflux disease. Dig Dis Sci 1997; 42:1362-1369.

29. Adachi K, Ono M, Kawamura A, Yuki M, Fujishiro H, Kinoshita Y. Nizatidine and cisapride enhance salivary secretion in humans. Aliment Pharmacol Ther 2002;16:297-301.

30. Besherdas K, Leahy A, Mason I, Harbord M, Epstein O. The effect of cisapride on dyspepsia symptoms and the electrogastrogram in patients with non-ulcer dyspepsia. Aliment Pharmacol Ther 1998;12: 755-759. 